\title{
Optimal values of seismic design coefficients considering variations of indirect economic losses
}

\author{
J. García-Pérez \\ Instituto de Ingeniería, Mecánica Aplicada, \\ Universidad Nacional Autónoma de México, Mexico
}

\begin{abstract}
In order to attain optimum design values in the optimization process, it is necessary to have both initial cost functions and losses due to earthquakes. These costs are described in terms of design parameters, usually the seismic design coefficient or the vibration period. Among the losses caused by earthquakes, there are indirect economic losses called intangibles which may involve human lives. Here we compute a lower limit to the social value of an anonymous life, in the sense of how much society is willing to invest in order to preserve a human life. The human capital point of view is adopted, and it is assumed that the value of human life would be the expected present value of the income that a person would have earned in the event that he/she had lived. Then we resort to the classical reliability theory and use concepts such as probability of failure, reliability function, hazard function, and their relationships. In order to explore the influence of intangibles, optimum design coefficients are computed for a specific site considering that earthquake arrival times constitute a Poisson process. The results show that the influence of indirect economic losses in the computation of seismic design coefficients does make a difference and should not be ignored or assigned arbitrarily.

Keywords: optimization, intangibles, total cost, structural reliability, utility.
\end{abstract}

\section{Introduction}

In order to make optimum decisions in earthquake engineering, we must quantify the consequences of our decisions. In this sense some techniques have been developed that allow us to obtain the optimum solution in rational decision- 
making, as long as we know the relationships between utility and benefits. In many problems the magnitude of benefits and losses are small enough to assume that utility is a linear function thereof. But very often, when intangibles such as human lives are involved, losses are so high that this hypothesis is not valid, and it is necessary to define the shapes of the utility functions (Rosenblueth [1]). Before assigning utilities we must decide whose utility is of concern, whether it is the investor, client or society. In fact the utility should consider all these subjects. Assigning utilities to intangibles presents a challenge. One way to solve the problem is by answering the question how much a person is willing to pay to obtain a benefit or to avoid a loss. Instead of asking how much the value of human life is, we must ask how much society is willing to pay to save a human life. Some early studies on the topic have suggested that society is willing to pay around the expected present value of the individual's income. Thus we begin this paper by discussing the optimization problem and by examining the expected present value of costs involved in the process, namely, the initial cost and the direct material and indirect economic losses. Then intangibles are analyzed considering the age of individuals as a main variable, because valuating intangibles as human life independent of age would not take into account the length of time that a life is extended. Moreover, age is a more relevant variable than the number of lives saved.

\section{Optimization}

When we try to design a structural system or component characterized by a design parameter vector $c$, the following objective function may be used, $v(c)=a(c)-u(c)-v(c)$ (Rosenblueth [2], Esteva [3]), where $a(c)$ is the benefit from the existence of the structure, $u(c)$ is the initial cost and $v(c)$ the expected damage cost. All these quantities represent expected values, and they will be measured in monetary units without loss of generality. In all cases in which failure of the system does not entail that the system no longer generates benefits, the problem of making maximum $a(c)-u(c)-v(c)$ is reduced to making minimum $u(c)+v(c)$ and $v(c)$ is a sum including the consequences of all failures of the system.

It is true that this economic model of the design process is not complete in the sense that it does not include either the design cost or the studies that this design requires. Furthermore, choosing the expected utility as the objective function deserves some comments. First, each participating party (society, investor, and client) may have different opinions regarding what the expected benefit is from a building. Thus, they will value the costs and losses differently. Moreover, each party will have a maximum amount of damage that it is willing to risk. One possible way to overcome this is by selecting a function that takes into account these concepts with appropriate weights, and other possibility is by representing the complete model by a decision tree, which shows the decisions that the designer can make, and the events that can happen. 


\section{Expected present values of costs}

\subsection{Initial cost}

A previously published paper (García-Pérez [4]) concludes that it is reasonable to adopt the following relationship between the initial cost $u$, and the seismic design coefficient $c$.

$$
u= \begin{cases}C & \text { if } c \leq c_{0} \\ {\left[1+\alpha_{2}\left(c-c_{0}\right)^{\alpha_{3}}\right] C} & \text { if } c>c_{0}\end{cases}
$$

where $c_{0}$ is the value of $c$ that the structure resists without being specifically designed against earthquakes, $C$ is the construction cost when it is not designed against earthquakes, $\alpha_{2}$ and $\alpha_{3}$ are constants depending on the height of the structure and the kind of soil where it is built.

\subsection{Direct material and indirect economic losses}

Let $D_{z}$ be the direct material loss (structural and nonstructural damage, and contents) due to damage to the building itself when subjected to an intensity $z$ and let $\zeta=z / c$. When $\zeta$ tends to zero $D_{z}$ should tend to zero. This comes from a common observation that very small earthquakes do not cause damage, regardless of how many. $D_{z}$ must be a monotonically increasing function of $\zeta$. We will take $D_{z}=u \xi(\zeta)$. The function $\xi(\zeta)$ must increase with $z$, thereby decreasing as $c$ increases so that $\lim _{z \rightarrow 0} \xi=0$ and $\lim _{z \rightarrow \infty} \xi=1$. Furthermore, it must tend very fast to zero when $\mathrm{z}$ tends to zero because we know that earthquakes of low intensity do not cause any damage. We take $\xi(\zeta): \xi(\zeta)=$ $0.025 \zeta^{6}-0.015 \zeta^{9}$ if $\zeta \leq 1$, and $\xi(\zeta)=\left(0.188+\zeta^{1.8}\right) /\left(117.8+\zeta^{1.8}\right)$ if $\zeta>1$.

In addition to the direct material loss $D_{z}$, there are noneconomic and indirect economic losses. These must be insignificant when $\xi(\zeta)$ is small because then the content of buildings does not suffer practically any damage, and they must tend to a higher quantity than $D_{z}=u \xi(\zeta)$ when it is large, because then we are dealing with buildings that suffer collapse, usually causing almost total loss of their content, loss of intangibles such as human lives and disruption of the economy in the affected area. We must include all the seismic losses caused by an earthquake of intensity $z$ by letting this loss be $L_{z}=u \xi(z / c)[1+b \xi(z / c)]$, where $b$ is a coefficient that depends on the intended use of the building.

\subsection{Seismicity}

We assume that the arrival times of earthquakes follow a Poisson process, and that the exceedance rates can be written as $\lambda(M)=\alpha_{1}\left(e^{-\beta M}-e^{-\beta M_{u}}\right)$ according to Cornell and Vanmarcke [5]. Here $\lambda(M)$ is the annual mean rate of events greater than or equal to $M, M_{u}$ is the maximum value of $M$ that can be generated in the province, and $\alpha_{1}$ and $\beta$ are constants. If linear conditions prevail 
we may associate the magnitude with the intensity by $z=H e^{\beta^{\prime} M}$, where $H$ is a function of the coordinates of the focus or rupture area and of the site of interest, and $\beta^{\prime}$ is a coefficient. Intensity can be the peak ground acceleration, spectral ordinates of systems of linear behavior with specific fundamental period of vibration and damping, or the demand of a base shear coefficient. For large earthquakes near a source, the intensity $z$ does not increase in the same proportion with $M$ as it does for greater distances. Consequently, there is a saturation phenomenon. In this case, we assume that at a given exceedance rate all values of $z$ duplicate except $z_{m}$, the maximum intensity that can occur at the site of interest. By combining $\lambda(M)$ and $H$ for each source, and integrating the contribution of all sources in order to get the total exceedance of $z$, we obtain a sum of functions. We assume that this sum can be written as: $\lambda(z)=\alpha_{4}\left(z^{-\alpha_{5}}-\right.$ $z_{m}^{-\alpha_{5}}$ ), where $\alpha_{4}$ and $\alpha_{5}$ are constants.

\subsection{Expected present value of all losses}

Let $-d \lambda / d z=\alpha_{4} \alpha_{5} z^{-\alpha_{5}-1}$ denote the density of occurrence of earthquakes with intensity $z, \lambda=\lambda(z)$ the exceedance rate of $z$. If the earthquake arrival times constitute a Poisson process, and we assume that the original condition is restored to the structure after each earthquake, and the discount rate $\gamma$ is independent of time and the expected cost of damage and failure per unit time is

$$
d_{0}=\int_{0}^{\infty}-\frac{d \lambda}{d z} L_{z}
$$

(Rosenblueth [6]), which is constant with time $t$, then the expected present value of all seismic losses becomes

$$
v=\int_{0}^{\infty} d_{0} e^{-\gamma t} d t
$$

and after substituting all variables

$$
v=\frac{\alpha_{4} \alpha_{5} u}{\gamma} \int_{0}^{z_{m}} \frac{\xi(z / c)[1+b \xi(z / c)]}{z^{\alpha_{5}+1}} d z
$$

It is advisable to write $\zeta_{m}=z_{m} / c$ in the above equation and integrate with respect to $\zeta$ rather than with respect to $z$. Then we get (García-Pérez, et al. [7])

$$
v=\frac{\alpha_{4} \alpha_{5} u}{\gamma^{\alpha_{5}}} \int_{0}^{\zeta_{m}} \frac{\xi(\zeta)[1+b \xi(\zeta)]}{\zeta^{\alpha_{5}+1}} d_{\zeta}
$$

\section{Intangibles}

Among losses caused by earthquakes, there are concepts called intangibles which may involve the human life. The meaning that we will give here to this intangible is not in the sense of responding to the question of how much a human 
life costs (because we know that the answer can be that it is priceless, infinite and beyond measure), but in the sense of how much society is willing to invest in order to preserve a life. It is undeniable that every time that we receive a supplementary wage for doing a job in which we are at risk, no matter how small, and every time that we decide how safe to make a civil structure, we are in fact assigning values to human lives.

\subsection{Approaches}

Some approaches historically used to deal with the value of human life are roughly: the human capital approach, consumptions and their variations, per capita gross domestic product, and willingness to pay (Linnerooth [8]).

The first studies on the value of human life adopt the human capital point of view, which sustains that the value of human life is the sum of income that the person has received for his/her job if he/she had not passed away prematurely. This approach has many drawbacks. It assigns zero values to those who do not earn money as children and elderly people. Thus, the main objection to this approach is that it only sees the problem from an economic point of view. In trying to overcome some of the objections found in the income criterion, the consumption criterion was proposed. In this criterion, the value of human life would be the expected present value of consumption that a person would have been attained in the event that he/she had lived. In order to compute the human life value we just replace earnings for consumptions. The consumption criterion is wrong in considering that everything consumed produces comfort. In the gross domestic product approach, an anonymous life is valued as the expected present value of a person's contribution to GDP during the rest of his/her life. The equations applied to the aforementioned methods are still applicable by replacing income or consumption with GDP. On the other hand, the willingness-to-pay approach specifically requires the use of utility curves in terms of the fortune and income of the persons under study. These curves must satisfy different conditions. In this approach utility is the logical measure of the intensity of preferences that satisfy the axiom of von Neumann and Morgenstern [9]. Strictly speaking, a more accurate approach to compute the value of human life should be based on utility curves, as well as on consideration of ethics concepts (Rosenblueth [11]). Nathwani et al. [10] have suggested the maximization of a social indicator of the quality of life as decision-making criterion, and Ditlevsen [12] has defined this index in a slightly more general way.

The criteria proposed in the literature to quantify the value of human life lead to such different results that it lacks of a reliable criterion. In this study, we will use the capital approach just in order to establish a lower limit to the social value of an anonymous life, and to see its influence in calculating optimum seismic design coefficients in earthquake engineering. 


\subsection{Reliability theory}

In the classical reliability theory, we have concepts such as probability of failure, reliability function, hazard function, and their relationships. We will review them hereinbelow.

The probability that a structure will fail during a period of time $t$ is given by $P[T \leq t]=F_{T}(t)=1-R_{T}(t)$, where $F_{T}()$ is the cumulative distribution function of $T . R_{T}()$ is the reliability or survival function expressed in terms of time, that is, the probability that the structure is still working at time $t, P[T>t]$. The probability density function (pdf) of the design life is given by $f_{T}(t)=$ $d\left[F_{T}(t)\right] / d t$, which is known as the unconditional failure rate, since it reflects the probability of failure in the time interval $t$ to $t+d t$ as $d t \rightarrow 0$.

The hazard function $h_{T}(t)$, also known as age specific failure rate or conditional failure rate, expresses the likelihood of failure in the time interval $t$ to $t+d t$ as $d t \rightarrow 0$, given that failure has not occurred prior to time $t$, that is: $h_{T}(t)=f_{T}(t) / R_{T}(t)$. The hazard function is not a pdf, though for a fixed time we can define the failure time density (lifetime density) conditional on survival to this fixed time. Thus $h_{T}(t) \cdot d t$ might be thought of as the instantaneous probability of failure at $t$, given survival to $t$.

A useful result is that the reliability function $R_{T}()$ may be expressed in terms of the hazard function $h_{T}()$ as: $R_{T}(t)=\exp \left[-\int_{0}^{t} h(u) d u\right]=\exp [-H(t)]$, where $H(t)$ is the cumulative hazard function.

\subsection{Analogy to social value}

According to the theory described, we make the following analogy. We call $F_{T}(t)=1-R_{T}(t)$ the probability that a person is dead prior to instant $t, R_{T}()$ the probability that a person is alive at time $t, f_{T}(t)$ the probability density function of the remaining lifetime, $h_{T}(t)$ the mortality rate taken as the conditional pdf of the remaining lifetime, given that a person is alive at instant $t$.

Now let $W(t)$ be the person's income per unit time, $t$ the time measured from the instant in which the life of a person is valuated, and $\gamma$ the discount rate. Thus the expected present value of a person's income during the rest of her life is given by: $L(t)=\int_{0}^{\infty} R_{T}(t) W(t) \exp (-\gamma t) d t$, which can be equated to the social value of a person's life.

\section{Applications}

\subsection{Lower limit to social value}

The data in terms of age of per capita income and mortality rates were taken from Mexico's Census Bureau (INEGI-2008) [13]. A Gaussian curve given by the following expression $W(t)=7.784 \exp \left[-(t-43.13)^{2} / 2(15.91)^{2}\right]$ and an exponential $h(t)=0.007126 \exp [0.0507 t]$ were used to fit the data as shown in figures 1 and 2, respectively. 


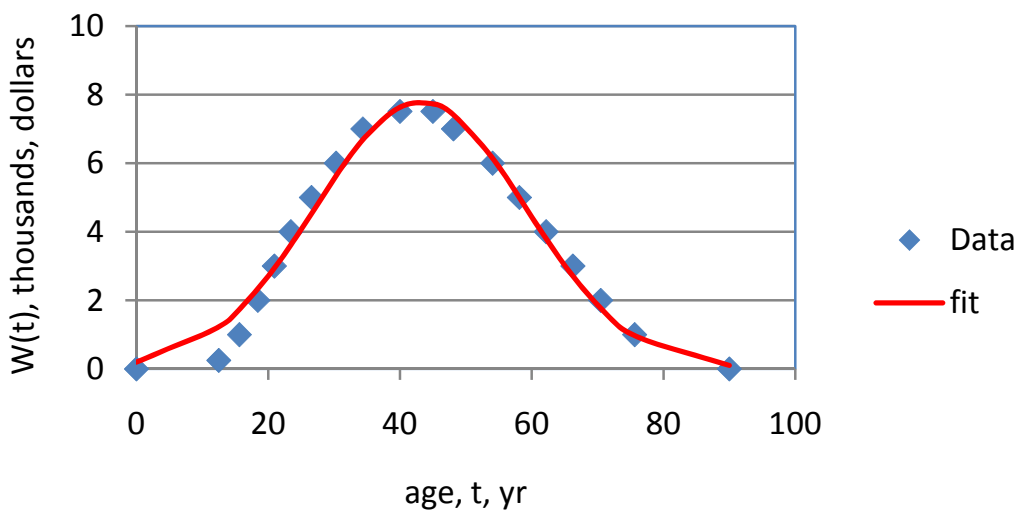

Figure 1: $\quad$ Per capita income in terms of age.

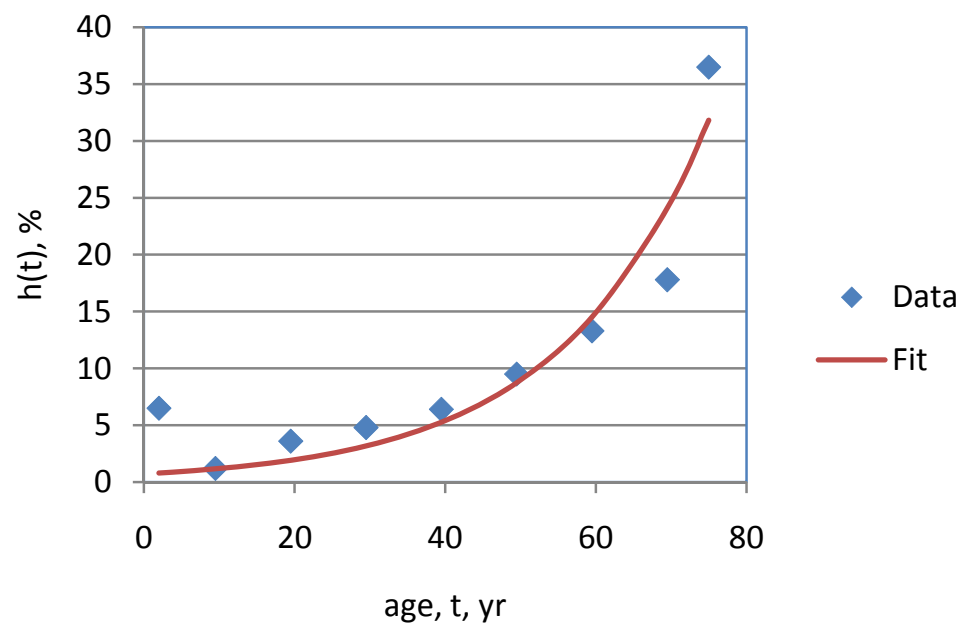

Figure 2: $\quad$ Mortality rates in terms of age.

After making substitutions, we compute $\mathrm{R}(\mathrm{t})$ as: $R(t)=8.95 \exp [-0.14(\exp (0.0507 t)]$. Figure 3 shows the results of the expected present value of a person's income using the approach developed. The form of the equation defining $L(t)$ assumes that it is updated at a continuous interest rate $\gamma=0.05$, which is more realistic than assuming that the interests are only generated or received once a year at a rate $\gamma$. If we assume that longevity is independent of income, with an average value of 3.43, which is evidently not rigorous (but sometimes is used as an approximation), we find that the results are overestimated at a little more than double. 


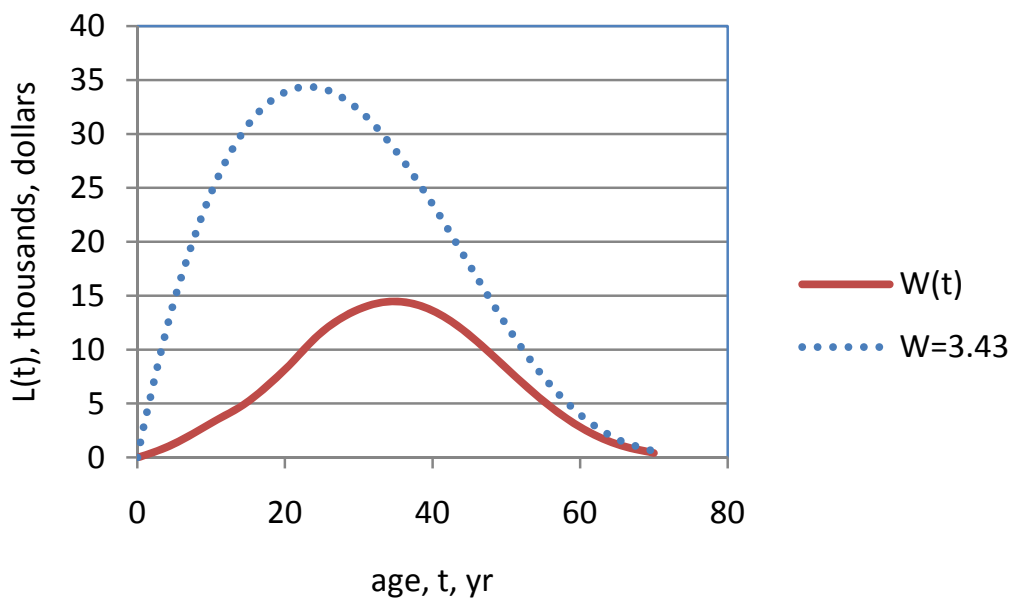

Figure 3: $\quad$ Expected present value of a person's income.

\subsection{Optimum seismic design coefficients}

In the development of construction codes, the value of intangibles should be taken into account for establishing safety levels. Such values will not appear in the codes, but the safety factors corresponding to each level and to the classification of the structures in safety levels in accordance with the use assigned. One way to take into account intangibles is to implicitly include them

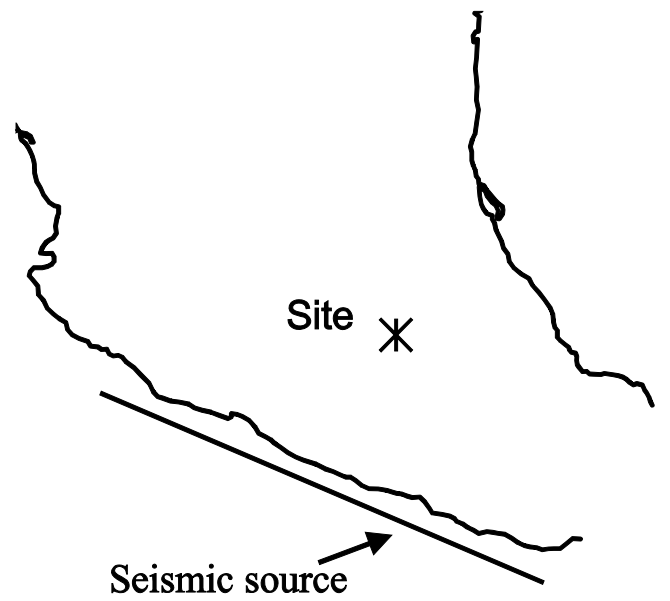

Figure 4: $\quad$ Site under study. 
in losses. In our case, we will compute the seismic optimum design coefficients by minimizing the total expected cost given by adding eqns (1) and (5) where the quadratic term measures the losses of content of the structures experiencing damages, as well as other costs to society due to earthquakes. In order to illustrate the influence of intangibles in computing optimum values, we will oversimplify the calculation by considering different values of coefficient $b$.

The optimum seismic design coefficient is computed for a site $400 \mathrm{~km}$ away from the seismic source, as shown in figure 4 . The values of the variables used are as follows: $\alpha_{2}=0.5, \alpha_{3}=1.2, c_{0}=0.1, \alpha_{4}=0.0005, \alpha_{5}=3.3, \gamma=$ $0.0686, b=1,12$. For the two values of coefficient $b$, there is a variation of four percent.

\section{Concluding remarks}

In the process of finding optimum values, it is relevant to include losses of intangibles. One of them is the cost that society is willing to incur in order to preserve a life. In this paper, we have used the human capital approach in order to establish a lower limit of such a value. Then we explore the influence of intangibles in computing optimum values. The most obvious objection to the human capital approach is noting that the job market is never perfect. And it has never been perfect. Adopting the hypothesis of its perfection seriously distorts the results. Thus, if we are interested in the value of life either for society or for the owner of that life; the human capital criterion should be discarded despite its extensive usage. We know that the value resulting from the human capital approach underestimates the value that should be assigned to life, because it considers the value of the money received while being alive with certain approximations, but not all the value of being alive. It also leaves out the value of the pleasure of living, as well as emotional and cultural values. However, we can use this value as a lower limit. Approaches such as the willingness-to-pay and ethics deserve some attention in order to establish a rational basis to account for the cost of preserving lives. The form of the willingness-to-pay approach must be based on utility curves which consider the quality of life at different ages, as well as societal impacts caused by deaths.

\section{References}

[1] Rosenblueth, E., Safety and structural design (Chapter 7). Reinforced Concrete Engineering, ed. Boris Bresler, Vol 1, John Wiley \& Sons, New York, pp. 407-516, 1974.

[2] Rosenblueth, E., Closure to probabilistic design to resist earthquakes. Journal of the Engineering Mechanics Div. Proc. ASCE, 90, EM5, 1964.

[3] Esteva, L., Bases para la formulación de diseño sísmico, PhD thesis, Faculty of Engineering, UNAM, 1968.

[4] García-Pérez, J., Seismic zoning for initial and total-cost minimization, Earthquake Engineering \& Structural Dynamics, 29, pp. 847-65, 2000. 
[5] Cornell, C.A., \& Vanmarcke, E., The major influences on seismic risk, IV WCEE, pp. 169-183, 1969.

[6] Rosenblueth, E., Optimum design for infrequent disturbances, ASCE, Journal of the Structural Division, 102 (ST109), pp. 1807-25, 1976.

[7] García-Pérez, J., Castellanos F., \& Díaz, O., Occupancy importance factor in earthquake engineering, Engineering Structures, 27, pp. 1625-32, 2005.

[8] Linnerooth, J., The value of human life: a review of the models, Economic Inquiry, 17, pp. 52-74, 1979.

[9] von Neumann, J., \& Morgenstern, O., Theory of games and economic behavior, Princeton University Press, Princeton, New Jersey, 1943.

[10] Nathwani, J.S., Lind, N.C., \& Pandey, M.D., Affordable Safety by choice: The Life Quality Method, Institute for Risk Research, University of Waterloo, Waterloo, Canada, 1997.

[11] Rosenblueth, E., What should we do with structural reliabilities, Reliability and risk analysis in civil engineering, Proc. Fifth International Conference on Applications of Statistics and Probability in Soil and Structural Engineering, Waterloo, Ontario, pp. 24-34, may, 1987.

[12] Ditlevsen, O., Decision modeling and acceptance criteria, Structural Safety, 25, pp. 165-91, 2003.

[13] INEGI, Mexico's Census Bureau. http://www.inegi.org.mx/ 